\title{
Stage I Choroidal and Ciliary Body Melanoma AJCC v8
}

National Cancer Institute

\section{Source}

National Cancer Institute. Stage I Choroidal and Ciliary Body Melanoma A/CC v8. NCI

Thesaurus. Code C140660.

Stage I includes: T1a, N0, M0. T1a: Tumor size category 1 without ciliary body involvement and extraocular extension. N0: No regional lymph node metastasis. MO: No distant metastasis. (AJCC 8th ed.) 